\title{
Photodecomposition of o-phthaldialdehyde-derivatized amino acids by the photodiode array detector during their high-performance liquid chromatographic analysis
}

\author{
Sándor Kéki ${ }^{1}$, János Török ${ }^{1}$, Bernadett Biri ${ }^{1}$, Judit Zsuga ${ }^{2,4}$, Rudolf Gesztelyi ${ }^{3}$ \\ Dániel Bereczki ${ }^{4}$, Miklós Zsuga ${ }^{1 *}$ \\ ${ }^{1}$ Department of Applied Chemistry, University of Debrecen, H-4012 Debrecen, Egyetem tér \\ Hungary \\ ${ }^{2}$ Department of Pharmacology and Pharmacotherapy, University of Debrecen, $\mathrm{H}-4012$ \\ Debrecen, Hungary \\ ${ }^{3}$ Departments of Pharmacology and Pharmacodynamics, University of Debrecen, $\mathrm{H}-4012$ \\ Debrecen, Hungary \\ ${ }^{4}$ Department of Neurology, University of Debrecen, H-4012 Debrecen, Nagyerdei krt 98, \\ Hungary
}

\begin{abstract}
During the high-performance liquid chromatographic (HPLC) analysis of o-phthaldialdehyde (OPA)-derivatized amino acids such as arginine and homoarginine we observed that the response of the fluorescence detection (FLD) system is decreased when the photodiode array detection (DAD) system (located before FLD system) was simultaneously switched on. The decrease in the FLD response, i.e., the difference in the FLD peak area $(\Delta \mathrm{A})$ obtained with DAD modes off $\left(\mathrm{A}_{\text {off }}\right)$ and on $\left(\mathrm{A}_{\text {on }}\right)$ was dependent upon the flow rate, but the relative FLD response decrease $\left(\Delta \mathrm{A} / \mathrm{A}_{\text {off }}\right)$ was practically independent of the amount of analyte injected. For example, decreasing the flow rate from $1 \mathrm{~mL} / \mathrm{min}$ to $0.5 \mathrm{~mL} / \mathrm{min}$ resulted in the relative decrease of FLD response from $\sim 5 \%$ to $\sim 11 \%$. It was concluded that the DAD caused a photoinduced partial decomposition of the OPA-derivatized amino acids flowing through the cell. The photoinduced dissociation of OPA derivatives was independently supported by using off-line photodiode array spectrometric measurements with long and short irradiation pulses. Based on the experimental results, for description of the variation of FLD responses with the flow rate upon the irradiation by the DAD a simple mathematical model is proposed and reported.
\end{abstract}

Keywords: OPA-derivatized arginine and homoarginine, high-performance liquid chromatography, photodecomposition, UV and fluorescence detection 


\section{Introduction}

Determinations of the amino acid levels in biological samples e.g. from human organisms may provide clues for understanding and prediction of the evolution of several disorders. Unusual variation in these concentrations may reflect to the presence and/or onset of serious disorders. For example, changes in the asymmetric dimethylarginine (ADMA), symmetric dimethylarginine (SDMA) and L-Arginin (Arg) levels in the serum or in the plasma are indicative of the increased risk for coronary heart diseases [1-3] and simultaneous evolution of atherosclerosis and insulin resistance [4-6]. In this respect, due to the possible connection between the corresponding amino acid levels in biological liquids and the disorders several methods have been developed for the qualitative and quantitative analysis of these compounds. Most of these methods include reversed phase high-performance liquid chromatographic (HPLC) separations of the pre-column-derivatized amino acids using UV and/or fluorescence and/or MS detections [7-12]. Because of the simplicity and reproducibility of the method and due to the UV and high fluorescent sensitivity of the derivatized amino acids, pre-column modification of these compounds with ophthaldialdehyde (OPA) [13] in the presence thiol derivatives such as 2-mercaptoethanol (2ME), 3-mercaptopropionic acid (3-MPA) or N-acetyl-L-cysteine (NAC) have become the most popular and widely accepted method for the determination of amino acids originating from biological samples [7-12]. The effect of the reaction conditions such as $\mathrm{pH}$, temperature and the ratio of reactant concentrations on the stability of a wide range of OPA-derivatized amino acids (in the presence of 2-ME and 3-MPA) have been thoroughly studied $[11,12]$. However, to our best knowledge no report on the stability of such compounds under HPLC conditions has been appeared.

In this article, we report a study on the change of fluorescence detection (FLD) system responses caused by the photodiode array detection (DAD) system as a function of the flow rate and a simple mathematical expression for the description of the above issues is also given.

\section{Experimental}

\section{1. Chemicals}

L-Argine (Arg) and L-homoarginine (HArg) hydrochloride, o-phthaldialdehyde (OPA), 3mercaptopropionic acid (3-MPA), boric acid, $\mathrm{NaOH}$, methanol and acetonitrile were purchased from Sigma-Aldrich (Steinheim, Germany) and were used without further 
purification. Ammonia solution and ammonium acetate were received from Reanal (Budapest, Hungary). All reagents used in this study were of highest purity available.

\subsection{Sample preparation, derivatization and chromatograpic separation}

The derivatization of Arg and HArg was achieved as described in Ref 12. Arg and HArg samples of $1000 \mu \mathrm{L}$ at a concentration of $6-20 \mu \mathrm{M}$ were mixed with $315 \mu \mathrm{L}$ of the orthophthaldialdehyde/3-mercaptopropionic acid reagent solution. The samples were incubated at $22{ }^{\circ} \mathrm{C}$ for 10 minutes then were cooled down to $5^{\circ} \mathrm{C}$. After $10 \mathrm{~min}$ incubation time aliquots of $10 \mu \mathrm{L}$ were injected into the chromatographic system which consisted of a Waters 2695 Separations Module equipped with thermostable autosampler $\left(5^{\circ} \mathrm{C}\right)$ and column module (35 ${ }^{\circ} \mathrm{C}$ ) and of a Waters 2475 Fluorescent and a Waters 2996 Photodiode-array detector. Separations were performed using an Agilent Zorbax SB-C18 (4.6 x 75 mm, $3.5 \mu \mathrm{m})$ column. Isocratic elution at a flow rate ranging from 0.5 to $1 \mathrm{~mL} / \mathrm{min}$ was applied using a mobile phase A consisting of $2.5 \mathrm{mM}$ ammonium acetate in water $(\mathrm{pH}=7.2)$ and phase $\mathrm{B}$ of ammonium acetate solution $(2.5 \mathrm{mM})$ and acetonitrile and methanol in 46/44/10 (v/v), respectively. Mobil phase A and B were mixed in a 4:1 volume ratio. Arg and HArg were detected at $\lambda_{\mathrm{ex}}=337 \mathrm{~nm} ; \lambda_{\mathrm{em}}=520 \mathrm{~nm}$. The precision of parallel injections was better than $0.5 \%$.

\subsection{Off-line spectrophotometric measurements}

The mixture of Arg and HArg at a concentration of $10 \mu \mathrm{M}(200 \mu \mathrm{L})$ was mixed with OPA/3MPA solutions of $400 \mu \mathrm{L}$ and diluted with the borate buffer to $1 \mathrm{~mL}$. After $10 \mathrm{~min}$ incubation time at ambient temperature the reaction mixture was placed into a quartz cuvette of optical path length of $1 \mathrm{~cm}$ and the absorbance at $230 \mathrm{~nm}$ was measured with a HP 8453 diode array spectrometer using cycling times of $30 \mathrm{~s}$ and $1 \mathrm{~s}$.

\section{Results and Discussion}

\subsection{Preliminary experiments}

During the HPLC analysis of the mixture of o-phthaldialdehyde (OPA)-derivatized Arg and HArg we observed that the response of the FLD decreased when the DAD that was located before FLD was also turned on. Albeit the relative decrease in the FLD response was not so high but it appeared significant. For instance, we observed a decrease in the FLD response by $-5 \%$ at a flow rate of $1 \mathrm{~mL} / \mathrm{min}$. We concluded that the decrease in the FLD response is due 
to the partial photodecomposition of these compounds caused by the light beam emitted from the DAD system. To support this conclusion, independent, off-line photometric experiments were conducted. In these experiments, we used diode array photometer and a reaction mixture similar to those applied for the HPLC experiments but the excess of the OPA/3mercaptopropionic acid (3-MPA) reagents to Arg and HArg was lowered in order to mimic the conditions occurring after LC separation of the OPA-derivatives from the unreacted OPA/ 3-MPA reagents. As an example, the result of these experiments is presented in Fig. 1.

Fig. 1.

Fig. 1. shows that by applying a cycling time of $30 \mathrm{~s}$ a relatively slow decrease in the absorbance at $230 \mathrm{~nm}$ can be observed. However, lowering the cycling time from $30 \mathrm{~s}$ to $1 \mathrm{~s}$, the absorbance decreases rapidly. This observation indicates and supports that photodecomposition of the OPA-derivatized Arg and HArg takes place upon the effect of light emanating, most probably, from the UV lamp of the photometer. On the other hand, under HPLC conditions it was found that the relative change in the FLD response caused by DAD system was depended on the flow rate. For example, at flow rates of $1,0.75$ and 0.5 $\mathrm{mL} / \mathrm{min}$ the relative decreases in the FLD responses were found to be 5.1, 7.2 and $11.2 \%$, respectively. To explain the FLD response change with the flow rate we took into account the considerations appended in the next section.

\section{2. Interpretation of the response change with the flow rate}

Based on the Lambert-Beer law it can be shown that the concentration of a photoactive analyte, $C(t)$ upon the irradiation of incident light with intensity, $I_{o}$ (photons/s) changes with time, $t$ according to Eq. 1 .

$$
C(t)=C_{o} \exp \left[-\frac{I_{o} \phi \gamma}{V_{\text {cell }} N_{A}} t\right]
$$

where $\gamma=\varepsilon L(\varepsilon=$ molar absorption coefficient at a given wavelength and $L$ is the optical path length); $V_{\text {cell }}$ and $N_{A}$ are the illuminated volume and the Avogadro-number, respectively. $C_{o}$ is the initial concentration of the analyte and $\phi$ is the quantum yield. 
However, under chromatographic conditions the value of $C_{o}$ is not constant, it changes continuously as the analyte is flowing through the cell of the photodiode array detector. In a general case, the concentration distribution, i.e., the peak shape can be given by a modified Gaussian distribution e.g. using parabolic variance [14].

The average resident time $(\tau)$ of the analyte in a cell volume $V_{\text {cell }}$ at a flow rate $v_{f l}$ is given by Eq. 2.

$$
\tau=\frac{V_{c e l l}}{v_{f l}}
$$

Since the residence time $\tau$ is short compared to the width of the chromatographic peak, therefore, it represents only a short slice in the whole distribution. For instance, the peak width at half maximum is about $0.12 \mathrm{~min}$ for Arg and HArg and the average residence time $(\tau)$ is $0.008 \mathrm{~min}$ at a flow rate of $1 \mathrm{~mL} / \mathrm{min}\left(V_{\text {cell }}\right.$ is $\left.8 \mu \mathrm{L}\right)$. This means that the height of each slice, i.e., related to the instant concentration of the analyte will decrease by a factor of $\exp \left(I_{o} \phi \gamma \tau / V_{\text {cell }}\right)$ when the photodiode array detector is turned on as shown in Eq. 3,

$$
A_{\text {on }}=A_{\text {off }} \exp \left[-\frac{I_{o} \phi \gamma}{V_{\text {cell }} N_{A}} \tau\right]
$$

where $A_{o n}$ and $A_{o f f}$ are the areas of the chromatographic peaks (detected by the fluorescence detector) obtained in the absence and presence of working photodiode array detector, respectively.

By substituting Eq. 2 into Eq. 3, Eq. 4 can be derived.

$$
\operatorname{Ln}\left(A_{o f f} / A_{o n}\right)=\frac{I_{o} \phi \gamma}{v_{f l} N_{A}}
$$

Eq. 4 suggest that by plotting the $\operatorname{Ln}\left(A_{o f f} / A_{o n}\right)$ values against $1 / v_{f l}$ should yield a straight line starting from the origin. Plot of $\operatorname{Ln}\left(A_{o f f} / A_{o n}\right)$ versus $1 / v_{f l}$ is shown in Fig. 2.

Fig. 2. 
According to Fig. 2. Ln $\left(A_{\text {off }} / A_{\text {on }}\right)$ changes linearly with $1 / v_{f l}$. The slope and the intercept for Arg are 0.056 and 0.0056 , and for HArg 0.05 and 0.01 , respectively. Considering the scattering in the experimental data, the slopes determined are very close to zero which supports the validity of Eq. 4.

\section{Conclusions}

It was shown that the photodiode array detector induces photodecomposition of the OPAderivatized Arg and HArg derivatives. The photodecomposition was also supported by offline spectrophotometric measurements using short and long cycling times. It was further observed that the extent of photodecomposition of these compounds increases as the flow rate is decreased. Based on the Lambert-Beer law an expression describing the extent of photodecomposition against the flow rate was derived and proposed. It should be mentioned that when simultaneous detection of the compounds with DAD and FLD was conducted no deviation from the linear plot of the injected sample amount versus response may be observed as the response in that case also changes linearly with the injected amount. Moreover, the sensitivity decreases. As we observed for the OPA-derivatized Arg and HArg the decrease in sensitivity is about $11 \%$ at a flow rate of $0.5 \mathrm{~mL} / \mathrm{min}$, however, this effect can be more considerable for other amino acids derivatized with OPA that may undergo photodecomposition to a greater extent.

\section{Acknowledgment}

This work was financially supported by the grants Nos. K-62213 and MU-00204/2001 given by OTKA (National Scientific Research Fund, Hungary) and the grants RET-006/2004 and GVOP-3.2.1.-2004-04-0152/3.0. 


\section{References}

[1]. J.T. Kielstein, C. Zoccali, Am. J. Kidney Dis. 46 (2005) 186.

[2]. V.P. Valkonen, H. Paiva, J.T. Salonen, T.A. Lakka, T. Lehtimaki, J. Laakso, R. Laaksonen, Lancet 358 (2001) 2127.

[3]. H. Lenzen, D. Tsikas, R.H. Boger, Eur. J. Clin. Pharmacol. 62 (2006) 45.

[4]. J. Zsuga, J. Török, M.T. Magyar, A. Valikovics, R. Gesztelyi, Á. Lenkei, L. Csiba, S. Kéki, M. Zsuga, D. Bereczki, Metabolism, 56 (2007) 394.

[5]. J. Zsuga, R. Gesztelyi, J. Török, S. Kéki, D. Bereczki, Med. Hypothesis 65 (2005) 1091.

[6]. J. Zsuga, J. Török, M.T. Magyar, A. Valikovics, R. Gesztelyi, S. Kéki, L. Csiba, M. Zsuga, D. Bereczki, Cerebrovasc Dis. 23 (2007) 388.

[7]. A. Vasanits, D. Kutlán, P. Sass, I. Molnár-Perl, J. Chromatogr. A 870 (2000) 271.

[8]. Y. Mengerink, D. Kutlán, F. Tóth, A. Csámpai, I. Molnár-Perl, J. Chromatogr. A 949 (2002) 99.

[9]. J. Pi, Y. Kumagai, G. Sun, N. Shimojo, J. Chromatogr. B 742 (2000) 199.

[10]. T.A.C. Vermeij, P.M. Edelbroek, J. Chromatogr. B 716 (1998) 233.

[11]. I. Molnár-Perl, I. Bozor, J. Chromatogr. A 798 (1998) 37.

[12]. I. Molnár-Perl, A. Vasanits, J. Chromatogr. A 835 (1999) 73.

[13]. M. Roth, Anal. Chem. 43 (1971) 880.

[14]. J.J. Baeza-Baeza, M.C. García-Alvarez-Coque, J. Chromatogr. A 1022 (2004) 17. 


\section{Legends for the Figures}

Fig. 1.

Absorbance (at $230 \mathrm{~nm}$ ) versus time plot for the reaction of Arg and HArg with OPA/3-MPA recorded by photodiode array photometer at ambient temperature. Experimental conditions: to the aquaeos solution of $\operatorname{Arg}(10 \mu \mathrm{M})$ and HArg $(10 \mu \mathrm{M})$ of $2 \mathrm{~mL} 36 \mu \mathrm{L}$ OPA/3-MPA solution was added and incubated for $15 \mathrm{~min}$ at ambient temperature.

Fig. 2.

$\operatorname{Ln}\left(A_{\text {off }} / A_{\text {on }}\right)$ versus $1 / v_{f l}$ plot for the OPA-derivatized Arg and HArg. The values were obtained by averaging the $A_{\text {off }}$ and $A_{\text {on }}$ values in the injected concentration range of 6-10 $\mu \mathrm{M}$ for Arg and HArg. 
Fig. 1.

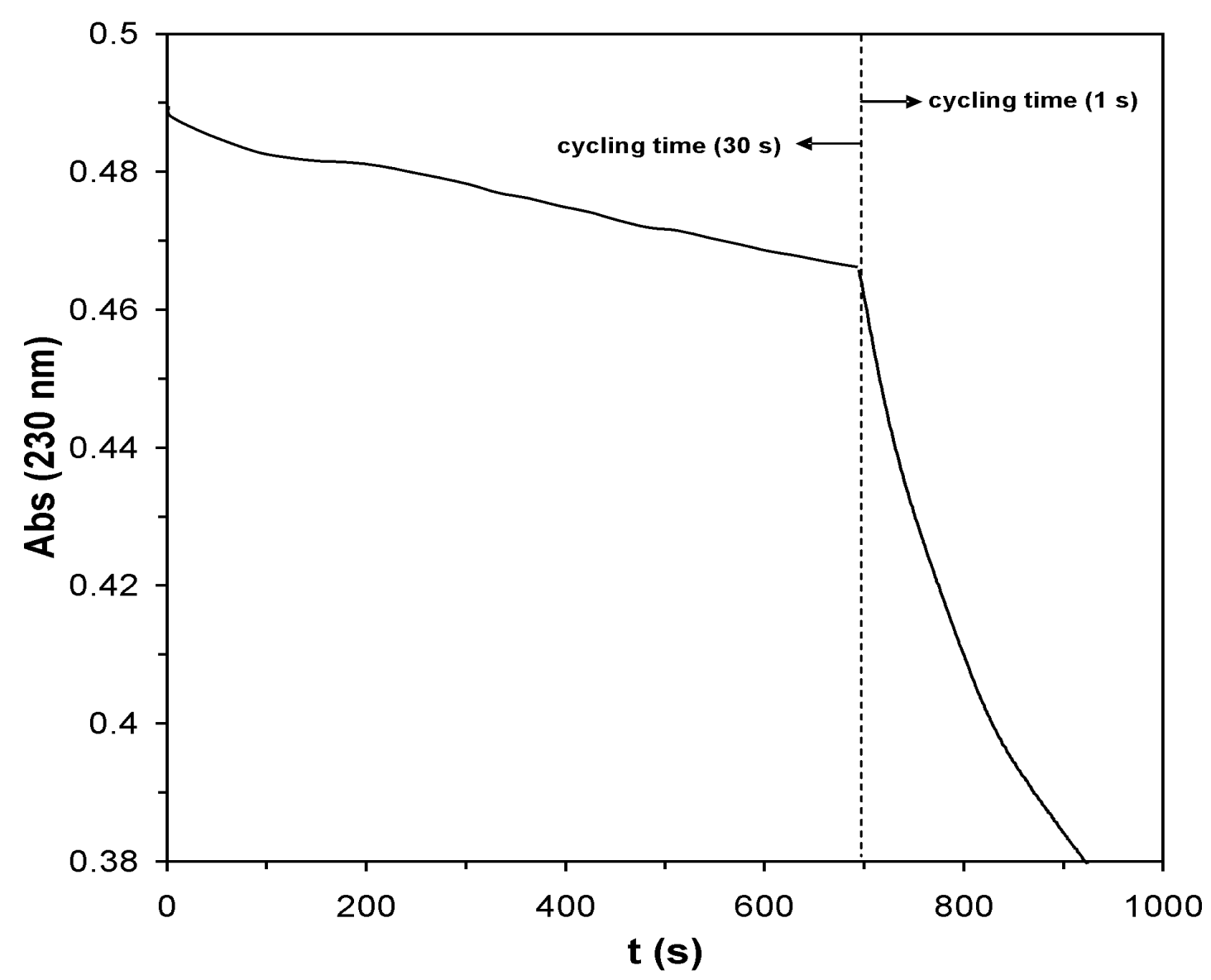


Fig. 2.

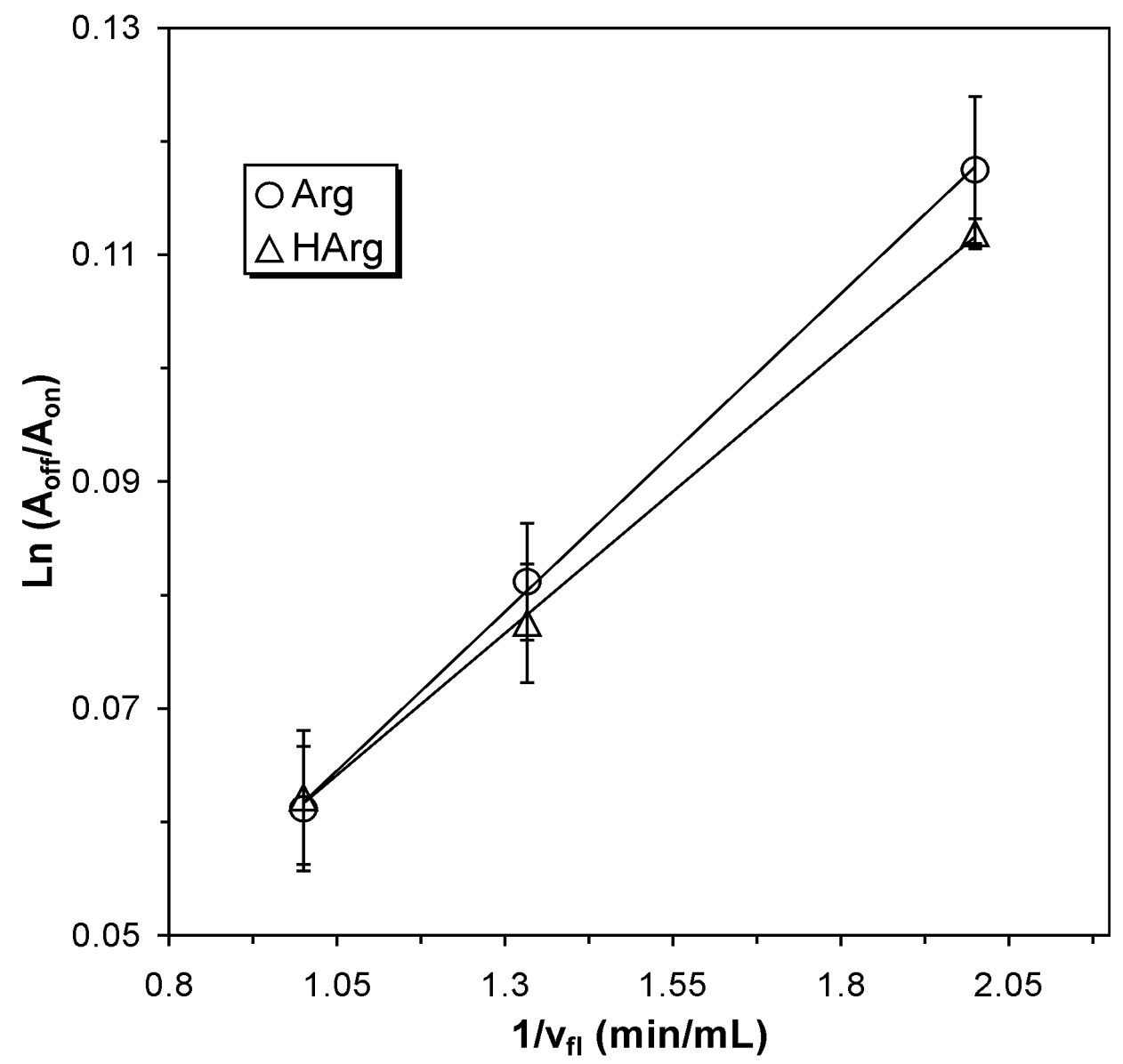

